\title{
A patient with acquired hemophilia A induced by clopidogrel
}

\author{
Hye Won Hwang, Jee Hyun Kong, Dong Wook Yu, Woo Taek Kim, Hyun Soo Kim, Chong In Lee \\ Division of Hematology-Oncology, Department of Medicine, Wonju Christian Hospital, Yonsei University College of Medicine, \\ Wonju, Korea
}

p-ISSN 1738-7949 / e-ISSN 2092-9129 http://dx.doi.org/10.5045/kjh.2012.47.1.80 Korean J Hematol 2012;47:80-2.

Received on July 29, 2011

Revised on September 20, 2011

Accepted on February 20, 2012

\author{
Correspondence to \\ Jee Hyun Kong, M.D. \\ Division of Hematology-Oncology, \\ Department of Medicine, Wonju Christian \\ Hospital, Yonsei University College of \\ Medicine, 162, Ilsan-dong, Wonju \\ 220-701, Korea \\ Tel: $+82-33-741-1201$ \\ Fax: +82-33-731-5884 \\ E-mail: kkongg@yonsei.ac.kr \\ (C) 2012 Korean Society of Hematology
}

Acquired hemophilia $\mathrm{A}(\mathrm{AHA})$ is a rare bleeding disorder caused by autoantibodies against factor VIII (FVIII). Treatment with clopidogrel is a cause of AHA, but its clinical course is unknown. Recently, we treated a 65-year-old man who was hospitalized for cerebellar infarction and had a prolonged activated partial thromboplastin time (aPTT) with soft tissue oozing after 3 weeks of clopidogrel use. We terminated clopidogrel administration and transfused the patient with fresh frozen plasma. However, the aPTT increased up to 98.8 seconds, and the FVIII and FVIII inhibitor levels were $<1 \%$ and 5.4 Bethesda units/mL, respectively. Clopidogrel-associated AHA was considered, and we began steroid treatment. Two months later, FVIII, FVIII inhibitor, and APTT values were normalized. No further bleeding or aPTT prolongation has been reported during the 2-year follow-up period. AHA should be considered in patients taking clopidogrel and experiencing bleeding, unless the platelet count and coagulation screen are normal.

Key Words Acquired hemophilia, Clopidogrel, Factor VIII, FVIII autoantibodies

\section{INTRODUCTION}

Acquired hemophilia A (AHA) is a rare bleeding disorder caused by autoantibodies against factor VIII (FVIII) [1]. It is diagnosed in patients without previous or familial histories of bleeding who have isolated prolongation of the activated partial thromboplastin time (aPTT) that cannot be corrected by mixing study and who have reduced FVIII levels as well as evidence of FVIII inhibitor activity [2]. FVIII autoantibodies occur in patients in the postpartum period and those with concomitant diseases or conditions (i.e., autoimmune disorders, malignancies, and drug abuse). However, up to $50 \%$ of cases develop without any relevant medical illness [3]. The mortality rate of AHA is as high as $16 \%$ [4].

Clopidogrel, an antiplatelet agent commonly used for coronary disease or cerebral vascular disease, is a cause of AHA [5]. However, only 5 cases of clopidogrel-related AHA have been described to date $[5,6]$. Because the exact natural history of clopidogrel-associated AHA is unknown, knowledge of its clinical features is important. The present report describes a patient with AHA associated with clopidogrel use who showed complete remission after steroid administration and discontinuation of clopidogrel.

\section{CASE REPORT}

A 65-year-old man was hospitalized for cerebellar infarction. He was referred to the hematology department due to prolonged aPTT. He had been administered aspirin, clopidogrel, and valproate for cerebellar infarction, a statin for hyperlipidemia, and cefoperazone, clindamycin, and teicoplanin for aspiration pneumonia. He had no previous or familial medical history of bleeding or coagulopathy and no clinical symptoms or signs of malignancy, antiphospholipid syndrome, or collagen vascular disease.

At admission, he was drowsy without clinical evidence of bleeding. He was intubated, and a tracheostomy was performed. Initial laboratory results showed normal complete blood cell counts, kidney and liver function, and coagulation parameters. However, 3 weeks after initiation of clopidogrel, blood oozed from his tracheostomy site, and his aPTT was 40.3 seconds (normal range, 27.9-37.8 seconds) (Fig. 1). Clopidogrel was considered the cause of bleeding, and its administration was discontinued. However, blood continued to ooze from his tracheostomy stoma, and his aPTT gradually rose up to 98.8 seconds (Fig. 1) without platelet or PT changes despite discontinuation of clopidogrel and

This is an Open Access article distributed under the terms of the Creative Commons Attribution Non-Commercial License (http://creativecommons.org/licenses/by-nc/3.0) which permits unrestricted non-commercial use, distribution, and reproduction in any medium, provided the original work is properly cited. 


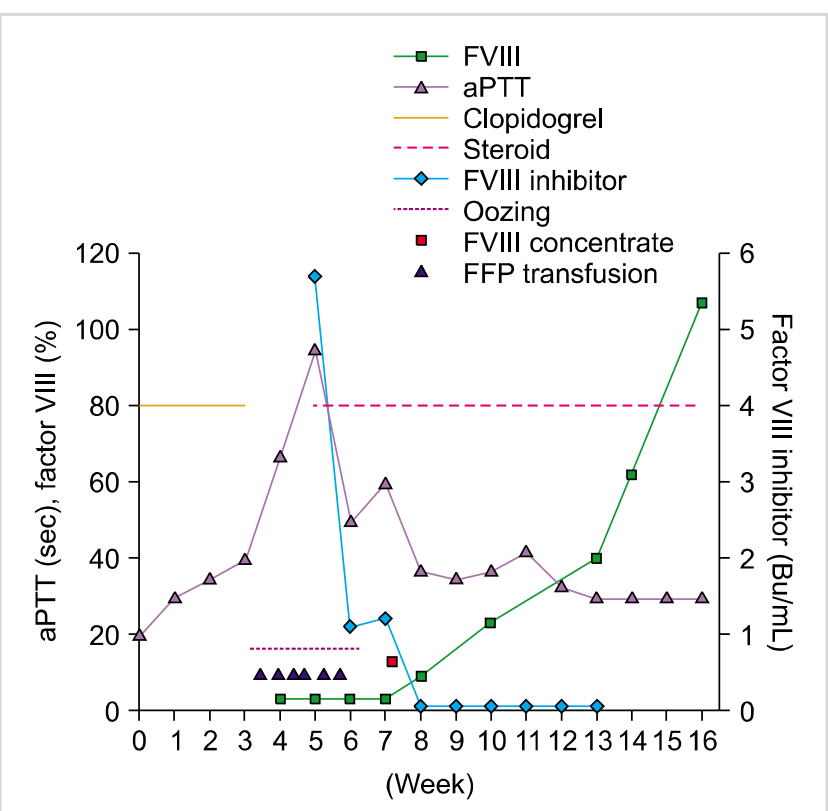

Fig. 1. Changes in aPTT, FVIII, and FVIII inhibitor levels as well as the use of blood products or medication after clopidogrel treatment. Abbreviations: aPTT, activated partial thromboplastin time; FVIII, factor VIII; FFP, fresh frozen plasma.

a transfusion with fresh frozen plasma. A mixing test did not correct the aPTT. Tests for lupus anticoagulant, anticardiolipin antibody, and antinuclear antibody were negative. His FVIII was below 1\% (reference range, 60-140\%), and his FVIII inhibitor titer was 5.4 Bethesda units $/ \mathrm{mL}$ (BU/mL) (Fig. 1).

We suspected clopidogrel-associated AHA and began steroid administration. Within a few days, the bleeding was controlled, and the patient's aPTT decreased to normal levels. However, 3 weeks after steroid use, active bleeding developed from his stoma. His aPTT was normal, and FVIII inhibitor was undetectable (Fig. 1). However, his FVIII was only 8\% (Fig. 1). We performed a left inferior thyroid arterial branch ligation and administered human FVIII concentrates (GreenMono, Greencross, Gyeonggi-do, Korea). Afterward, there were no further bleeding episodes, and his FVIII was normalized 2 months after steroid treatment. The patient was gradually tapered off steroids in 3 months. During the 2-year follow-up period, his aPTT remained within normal levels.

\section{DISCUSSION}

Clopidogrel is increasingly used as a first-line antiplatelet agent in patients with coronary artery disease, cerebral vascular disease, and peripheral vascular disease [7]. Clopidogrel is known to have adverse hematologic effects, such as neutropenia [8, 9], idiopathic immune thrombocytopenia [10], thrombotic thrombocytopenic purpura [11], and hemolytic uremic syndrome [12]. Recently, 5 cases of clopidogrel-associated AHA have been reported $[5,6]$.
In general, there is an equal distribution of AHA between the sexes. However, there is a female predominance in the younger age group because of an association with pregnancy, and males constitute the majority of patients over the age of 60 who develop AHA $[13,14]$. However, in 5 cases of clopidogrel-associated AHA, 4 were females, and all were over the age of 60 years (67-78 years) [6]. Nevertheless, a predominance of clopidogrel-associated AHA in females is difficult to conclude due to the rarity of the condition. It appears more likely to occur in older patients, who tend to have vascular diseases and, as a result, more commonly use clopidogrel. In the present case, soft-tissue bleeding and aPTT prolongation developed within 3 weeks after clopidogrel treatment. Because the patient was hospitalized during that episode, we were able to detect the abnormal aPTT earlier than in 2 previous cases, where AHA developed in 2-3 months after clopidogrel use [5].

In the literature, there are 34 cases of drug-induced AHA with FVIII inhibitor titers ranging from 1.6 to $250 \mathrm{BU} / \mathrm{mL}$ (average, 67.7 BU/mL) [6]. Those with clopidogrel-associated AHA had FVIII titers from 2.2 to 17 [6]. The present patient had a FVIII titer of $5.4 \mathrm{BU} / \mathrm{mL}$. However, according to that analysis [6], FVIII inhibitor titers were not well correlated with FVIII levels or clinical manifestation. In the present case, FVIII levels were normalized in about 7 weeks after the FVIII inhibitor titer was normalized. This discordance may arise from the different pattern of FVIII inactivation as a result of autoantibodies in AHA versus alloantibodies in congenital hemophilia [15]. In fact, whereas alloantibodies usually completely inactivate FVIII activity (type I kinetics), autoantibodies often incompletely inactivate FVIII activities (type II kinetics), and some residual FVIII activity remains in the patient's plasma [15]. Therefore, the Bethesda assay, which quantifies the in vitro inhibitor titer, may underestimate in vivo inhibitor potency in AHA [6].

There are no guidelines with respect to treatment for AHA, although its mortality rate is as high as $16 \%$ [4]. In over $80 \%$ of the cases, FVIII inhibitor disappeared after discontinuation of clopidogrel or after immunosuppressive therapy [6]. The present patient's FVIII was normalized after 7 weeks of steroid use. This is similar to 2 cases reported by Haj et al., in which FVIII returned to normal levels, and FVIII inhibitor was undetectable within 8 weeks of steroid treatment [5] and remained normal for 2 years. Therefore, bleeding tendencies in patients using clopidogrel should not be regarded as an antiplatelet effect of clopidogrel unless a platelet count and coagulation screen are normal. Immunosuppressive therapy should be initiated as soon as AHA is diagnosed.

\section{REFERENCES}

1. Green D, Lechner K. A survey of 215 non-hemophilic patients with inhibitors to factor VIII. Thromb Haemost 1981;45:200-3.

2. Delgado J, Jimenez-Yuste V, Hernandez-Navarro F, Villar A. Acquired haemophilia: review and meta-analysis focused on 
therapy and prognostic factors. Br J Haematol 2003;121:21-35.

3. Toschi V, Baudo F. Diagnosis, laboratory aspects and management of acquired hemophilia A. Intern Emerg Med 2010;5:325-33.

4. Zeitler H, Ulrich-Merzenich G, Hess L, et al. Treatment of acquired hemophilia by the Bonn-Malmo Protocol: documentation of an in vivo immunomodulating concept. Blood 2005; 105:2287-93.

5. Haj M, Dasani H, Kundu S, Mohite U, Collins PW. Acquired haemophilia A may be associated with clopidogrel. BMJ 2004; 329:323.

6. Franchini M, Capra F, Nicolini N, et al. Drug-induced anti-factor VIII antibodies: a systematic review. Med Sci Monit 2007;13: RA55-61.

7. Balamuthusamy S, Arora R. Hematologic adverse effects of clopidogrel. Am J Ther 2007;14:106-12.

8. Mehta SR, Yusuf S. The Clopidogrel in Unstable angina to prevent Recurrent Events (CURE) trial programme; rationale, design and baseline characteristics including a meta-analysis of the effects of thienopyridines in vascular disease. Eur Heart J 2000;21:2033-41.
9. A randomised, blinded, trial of clopidogrel versus aspirin in patients at risk of ischaemic events (CAPRIE). CAPRIE Steering Committee. Lancet 1996;348:1329-39.

10. Elmi F, Peacock T, Schiavone J. Isolated profound thrombocytopenia associated with clopidogrel. J Invasive Cardiol 2000;12: 532-5.

11. Bennett CL, Connors JM, Carwile JM, et al. Thrombotic thrombocytopenic purpura associated with clopidogrel. N Engl J Med 2000;342:1773-7.

12. Medina PJ, Sipols JM, George JN. Drug-associated thrombotic thrombocytopenic purpura-hemolytic uremic syndrome. Curr Opin Hematol 2001;8:286-93.

13. Cohen AJ, Kessler CM. Acquired inhibitors. Baillieres Clin Haematol 1996;9:331-54.

14. Hay CR. Acquired haemophilia. Baillieres Clin Haematol 1998;11: 287-303.

15. Green D, Blanc J, Foiles N. Spontaneous inhibitors of factor VIII: kinetics of inactivation of human and porcine factor VIII. J Lab Clin Med 1999;133:260-4. 